\title{
Dietary inflammatory index is associated with pain intensity and some components of quality of life in patients with knee osteoarthritis
}

\author{
Vahideh Toopchizadeh ${ }^{1}$, Neda Dolatkhah ${ }^{{ }^{*}}$ (D), Dawood Aghamohammadi ${ }^{2}$, Mahrokh Rasouli ${ }^{3}$ \\ and Maryam Hashemian ${ }^{4}$
}

\begin{abstract}
Objectives: We aim to measure dietary inflammatory index (DII) and its association with functional status, pain intensity and quality of life (QOL) in patients with knee osteoarthritis (KOA). Dietary information from 220 qualified patients with KOA was collected by a 168-item food frequency questionnaire. The functional status, pain intensity and QOL were evaluated by Western Ontario and McMaster Universities Osteoarthritis Index (WOMAC) questionnaire, Visual Analogue Scale (VAS) and SF36 questionnaire respectively.

Results: Linear regression analysis showed that the DII was significantly associated with VAS ( $p=0.040 ; \beta=0.151)$, and physical function $(p=0.039 ; \beta=-0.184)$, emotional wellbeing $(p=0.048 ; \beta=-0.158)$ and pain $(p=0.020$; $\beta=0.161)$ scales and physical health $(p=0.047 ; \beta=0.110)$ subscale of $Q O L$ after adjusting for age, sex, body mass index, and physical activity. There was no significant differences concerning WOMAC across the DII tertiles with and without adjustment to probable confounders ( $P_{\text {trend }}=0.091$ and 0.181 , respectively). After adjustment, a significantly increased severe pain odds was observed in the highest tertile of DII score in comparison with the lowest tertile (OR tertile $\left._{3 \text { vs. } 1}=1.55,95 \% \mathrm{Cl} 1.04-2.31 ; P_{\text {trend }}=0.04\right)$.
\end{abstract}

Keywords: Dietary Inflammatory Index, Osteoarthritis, Pain, Functional status, Quality of life

\section{Introduction}

Knee osteoarthritis (KOA) is a musculoskeletal disorder of an inflammatory nature that affects people around the world, causing pain, physical disability, and lowering the quality of life (QOL) [1]. Previous studies have shown that inflammation play a role in the pathogenesis and the development of osteoarthritis (OA) [2] and an important factor associated with the loss of cartilage and symptoms of the disease [3].

\footnotetext{
*Correspondence: neda_dolatkhah@yahoo.com; dolatkhahn@tbzmed.ac.ir 1 Physical Medicine and Rehabilitation Research Center, Aging Research Institute, Tabriz University of Medical Science, Azadi Ave., Tabriz, Iran Full list of author information is available at the end of the article
}

Various studies have shown that one's dietary habits play an important role in regulating chronic inflammation $[4,5]$ and demonstrate a "dietary basis" for the inflammatory presentation in rheumatic and musculoskeletal diseases, recommending an organized multidisciplinary approach to manage these conditions $[6,7]$. An ideal nutritional pattern is essential for regulating inflammation and oxidative stress, which are interrelated with the immune system [8]. The central conception of the relationship between dietary components and inflammatory and oxidative stress procedures has been well-recognized and corroborated, for instance, in the creation of the dietary inflammation index (DII). DII is a scoring system for measuring the potential of diet in inducing 
inflammation. DII validity regarding inflammatory markers such as C-reactive protein (CRP) and interleukin 6 has been shown in previous studies $[9,10]$. This indicates the capability of this indicator in determining the relationship between inflammatory compliance of diet and chronic disease $[11,12]$.

Considering the importance of the topic and rarity of studies in this area, especially in patients with KOA, this study was designed to firstly identify the DII in patients with KOA, and secondly, the association of DII with functional status and pain intensity and QOL in these patients.

\section{Main text \\ Procedure}

This descriptive cross-sectional study was conducted on 220 individuals with KOA during the March-September 2018. Participants were selected via proportional random sampling from the health centers of Tabriz City and then phoned, a summary explanation of the objectives and process of the research were described and, if they agreed to contribute in the study, they were requested to be present at the selected time in the center.

\section{Inclusion criteria}

Diagnosis of primary KOA defined by the American College of Rheumatology classification criteria [13], age 45 years old and over and no other inflammatory disease such as rheumatoid arthritis.

\section{Exclusion criteria}

Follow a special dietary pattern for medical or other reasons, subjects with neuropathy and sensory impairment, body mass index $(\mathrm{BMI}) \geq 35$, history of knee joint injection in the last 6 months or the use of corticosteroid medications for a recent month, people who are unable to cooperate in completing questionnaires and procedures for any reason.

\section{Assessment of dietary intake}

A validated 168 items semi-quantitative food frequency questionnaire (FFQ) [14] was used for examining participants' dietary intakes in previous 1 year. Each food and drink was coded and entered in Nutritionist IV software to assess the average daily intake of energy and nutrients including protein, carbohydrate, fat, saturated fats, monounsaturated fatty acids, polyunsaturated fatty acids, omega-3 fatty acids, and omega- 6 fatty acids, cholesterol, fiber, Vitamin A, Vitamin C, Vitamin D, Vitamin E, Vitamin B12, Vitamin B1, Vitamin B2, Vitamin B6, niacin, folate, beta-carotene, iron minerals, zinc, magnesium.

\section{Dietary Inflammatory Index (DII)}

Dietary intakes of 29 abovementioned food parameters were used to calculate the DII. In order to reduce between-subject variation, dietary intake was adjusted to the total daily energy intake using residual method [15]. The food information then was linked to the representative regional database, which estimates the global average intake of each nutrient with its standard deviation [16]. To calculate the subject's exposure relative to the standard global mean, the standardized meanings were subtracted from these values and divided into the corresponding standard deviations. The $\mathrm{z}$-scores obtained from this method turned into percentiles and doubled, and then " 1 " declined. Finally, the centralized percentiles for each parameter were multiplied by their related total inflammatory effect score and the total obtained values were added together to obtain an overall DII. $\mathrm{DII}=\mathrm{b} 1 * \mathrm{n} 1+\mathrm{b} 2 * \mathrm{n} 2 \ldots . . \mathrm{b} 29 * \mathrm{n} 29$, where $b$ is the literature-derived inflammatory effects score for each food parameter and $\mathrm{n}$ is the food parameter-related centered percentiles, which were achieved from the participants' dietary data. Additionally, we created groups based on the DII tertiles.

\section{Demographics characteristics}

For all participants, the demographic information was gathered via a general information questionnaire. Body weight and height were measured by digital scale (Seca, Hamburg, Germany) and a non-stretched tape measure (Seca, Hamburg, Germany), respectively. The Persian version of International Physical Activity Questionnaire (IPAQ) was applied to measure physical activity $[17,18]$.

\section{Visual analogue scale (VAS)}

VAS (0-10 $\mathrm{cm}$ pain scale: $0=$ none and $10=$ intolerable) was applied to measure the pain intensity in the participants. The validity of using this scale for assessing the pain intensity has been demonstrated in the studies [19].

\section{The Western Ontario and McMaster (WOMAC) index}

WOMAC index was applied to measure the functional status of participants by considering twenty-four items categorized into three subscales: pain (5 items), stiffness (2 items) and physical function (17 items) [20]. It is one of the frequently used tools to quantity the physical performance related with OA [21]. The WOMAC scores can be linearly changed to a 0-96 scale, with higher scores representing more severe symptoms and impaired function. The validity and reliability of the Persian version of WOMAC index have been established in studies [22]. 


\section{6-item short form health survey (SF-36)}

The Persian version SF36 was used to measure QOL in participants. The questionnaire has 36 questions, which assesses eight different areas of health with respect to the physical and emotional aspects. Likert scales and yes/no options are applied to evaluate function and well-being. Scales are standardized with a scoring process to achieve a total score fluctuating from zero to 100 . Its validity and reliability have been established in Iran [23].

\section{Statistical analysis}

The 168-item semi-quantitative FFQ, categorizes the food items into twenty-two groups. It has been recommended that the sample size for such studies to be considered as ten subjects per variable [24]. Consequently, the sample size was calculated as 220 subjects.

Normal distribution of variables was tested using Kolmogorov-Smirnov test. Linear regression and Chi square tests were applied to examine the trend of continuous and categorical variables across DII tertiles, respectively. The relationships between the DII score and pain intensity, functional disability and QOL were analyzed by linear regression analysis (Model 0 , unadjusted and Model I, adjusting for age, sex, physical activity and BMI. Odds ratio (ORs) and 95\% confidence interval (CI) of severe pain and disability across DII tertiles were measured by logistic regression analysis, whereas the lowest tertile of DII considered as the reference. The SPSS version 17.0 (IBM SPSS Statistics for Windows, Version 17.0. Armonk, NY: IBM Corp) has been used to analyze data.

\section{Results}

From 348 subjects who were screened, a total of 220 patients with KOA were qualified for inclusion in the investigation. Demographic characteristics of the participants according to the DII tertiles are shown in Table 1. There was a significant difference concerning the gender of patients across the DII tertiles. Most of men (32 out of 68) were in the top tertile of the DII, while most of women (56 out of 152) were in the first tertile of the DII $(p=0.033)$. Additionally, subjects in top tertile had significantly higher BMI than two other tertiles $(\mathrm{p}=0.032)$. There was no significant difference in other demographic variables across tertiles of DII.

Data on the pain intensity, functional status and scales and subscales of QOL in the patients across the DII tertiles are shown in Table 2. The finding of linear regression after adjusting for age, sex, BMI and physical activity showed that here were significant differences concerning pain intensity (VAS) and physical function (PF),

Table 1 General characteristics of patients with knee osteoarthritis across the DII tertiles

\begin{tabular}{|c|c|c|c|c|c|c|c|}
\hline \multirow[t]{2}{*}{ Variable } & \multicolumn{6}{|l|}{ DIl score } & \multirow[t]{2}{*}{ p value* } \\
\hline & $\begin{array}{l}\text { DII Score Tertile } 1 \\
(<-1.3) \\
(n=73)\end{array}$ & & $\begin{array}{l}\text { DII Score Tertile } 2 \\
(-1.3 \text { to } 4.5) \\
(n=73)\end{array}$ & & $\begin{array}{l}\text { DII Score Tertile } 3 \\
(>4.5) \\
(n=74)\end{array}$ & & \\
\hline Age (years) & $0.94^{*} \pm 57.85$ & & $1.17^{*} \pm 54.92$ & & $1.21^{*} \pm 57.91$ & & 0.091 \\
\hline $\mathrm{BMI}$ & $3.48^{*} \pm 36.41$ & & $0.54^{*} \pm 29.65$ & & $0.69^{*} \pm 29.66$ & & 0.032 \\
\hline \multicolumn{8}{|l|}{ Sex } \\
\hline Male & 17 & 25.00 & 19 & 27.94 & 32 & 47.06 & 0.033 \\
\hline Female & 56 & 36.84 & 54 & 35.53 & 42 & 27.63 & \\
\hline \multicolumn{8}{|l|}{ Education } \\
\hline Illiterate & 32 & 26.91 & 35 & 32.71 & 40 & 37.38 & 0.173 \\
\hline Low literate and Diploma & 31 & 43.66 & 22 & 30.99 & 18 & 25.35 & \\
\hline College & 10 & 23.81 & 16 & 38.10 & 16 & 38.10 & \\
\hline \multicolumn{8}{|l|}{ Occupation } \\
\hline Unemployed & 34 & 29.57 & 39 & 23.91 & 42 & 36.52 & 0.477 \\
\hline Employed & 23 & 32.86 & 25 & 35.71 & 22 & 31.43 & \\
\hline Retired & 16 & 45.71 & 9 & 25.71 & 10 & 28.57 & \\
\hline \multicolumn{8}{|l|}{ Physical activity } \\
\hline Low & 48 & 65.75 & 39 & 53.42 & 40 & 54.05 & 0.398 \\
\hline Middle & 20 & 27.39 & 21 & 28.76 & 18 & 24.32 & \\
\hline High & 5 & 6.84 & 13 & 17.80 & 16 & 21.62 & \\
\hline
\end{tabular}

Data are presented as mean \pm SD or $n(\%)$

Chi square test was applied for categorical variables; Linear regression was applied for continuous variables $B M I$ body mass index, DII dietary inflammatory index, yrs years

*p-value $<0.005$ were deemed significant 
Table 2 The differences on functional status, pain intensity and quality of life among DII score tertiles of patients with knee osteoarthritis

\begin{tabular}{|c|c|c|c|c|c|}
\hline \multirow[t]{2}{*}{ Variable } & \multicolumn{3}{|l|}{ DIl score } & \multirow[t]{2}{*}{$p$ for trend $d^{a}$} & \multirow[t]{2}{*}{$\mathrm{p}$ for trend $\mathrm{d}^{\mathrm{k}}$} \\
\hline & $\begin{array}{l}\text { DII Score Tertile } 1 \\
(<-1.3)(n=73)\end{array}$ & $\begin{array}{l}\text { DII Score Tertile } 2 \\
(-1.3 \text { to } 4.5)(n=73)\end{array}$ & $\begin{array}{l}\text { DII Score Tertile } 3 \\
(>4.5)(n=74)\end{array}$ & & \\
\hline VAS & $0.20 \pm 4$ & $0.01 \pm 6$ & $0.03 \pm 6$ & 0.001 & 0.012 \\
\hline WOMAC & $2.21 \pm 40$ & $2.32 \pm 44$ & $2.12 \pm 45$ & 0.181 & 0.091 \\
\hline \multicolumn{6}{|l|}{$\mathrm{QOL}$} \\
\hline PF & $3.80 \pm 55.54$ & $3.95 \pm 47.40$ & $3.71 \pm 41.23$ & 0.031 & 0.014 \\
\hline RP & $57.43 \pm 2.88$ & $3.31 \pm 50.51$ & $41.61 \pm 3.73$ & 0.004 & 0.010 \\
\hline RE & $3.73 \pm 35.14$ & $3.88 \pm 38.36$ & $3.78 \pm 30.59$ & 0.351 & 0.215 \\
\hline$E / F$ & $2.27 \pm 50.34$ & $2.33 \pm 49.38$ & $2.1 \pm 44.18$ & 0.111 & 0.085 \\
\hline EW & $1.92 \pm 44.99$ & $2.07 \pm 48.83$ & $1.70 \pm 44.43$ & 0.207 & 0.102 \\
\hline SF & $2.53 \pm 55.91$ & $2.65 \pm 53.42$ & $2.4 \pm 47.60$ & 0.061 & 0.049 \\
\hline$P$ & $2.67 \pm 64.73$ & $2.69 \pm 58.60$ & $2.6 \pm 54.52$ & 0.025 & 0.043 \\
\hline $\mathrm{GH}$ & $2.5 \pm 50.55$ & $2.91 \pm 47.47$ & $2.97 \pm 48.59$ & 0.737 & 0.126 \\
\hline $\mathrm{PH}$ & $228.29 \pm 7.58$ & $204.04 \pm 5.02$ & $186.98 \pm 4.18$ & 0.011 & 0.045 \\
\hline $\mathrm{MH}$ & $186.47 \pm 3.79$ & $190.03 \pm 8.61$ & $166.82 \pm 3.51$ & 0.517 & 0.147 \\
\hline
\end{tabular}

DIl dietary inflammatory index, EF energy/fatigue, EW emotional well-being, $G H$ general health, $M H$ mental health, $P$ pain, $P F$ physical function, $P H$ physical health, $Q O L$ quality of life, $R E$ role limitation due to emotional problems, $R P$ role limitation due to physical health, $S F$ social function, VAS, visual analogue scale, WOMAC,Western Ontario and McMaster Index

a Linear regression across DII score tertiles

b Linear regression across DII score tertiles with adjustment for age, sex, body mass index and physical activity

role limitation due to physical health (RP), social function $(\mathrm{SF})$ and pain $(\mathrm{P})$ scales and physical health $(\mathrm{PH})$ subscales of QOL across the DII tertiles ( $\mathrm{p}$ for trend of $0.012,0.014,0.010,0.049,0.043$ and 0.045 respectively). According to the adjusted standardized regression coefficients, higher DII scores were associated with higher pain intensity $(\beta=0.171)$ and lower PF $(\beta=-0.184)$, emotional well-being (EW) $(\beta=-0.158)$ and $P(\beta=-0.161)$ scales and PH ( $\beta=-0.142)$ subscales of QOL (Additional file 1).

Crude and adjusted odds ratios and 95\% confidence intervals for the association between DII and severe pain and disability according to tertiles of DII score in patients with $\mathrm{KOA}$ are presented as Table 3. After adjusting for some potential confounders, a significantly increased severe pain odds was observed in the highest tertile of

Table 3 Odds ratios and $\mathbf{9 5 \%}$ confidence intervals for the association between DII and severe pain and disability according to tertiles of DII score in patients with knee osteoarthritis

\begin{tabular}{|c|c|c|c|c|c|c|}
\hline & \multicolumn{3}{|l|}{ DIl score } & \multirow[t]{2}{*}{$p$ for trend $d^{a}$} & \multirow[t]{2}{*}{ Continuous OR } & \multirow[t]{2}{*}{$95 \% \mathrm{Cl}$} \\
\hline & $\begin{array}{l}\text { DII Score } \\
\text { Tertile } 1 \\
(<-1.3) \\
(n=73)\end{array}$ & $\begin{array}{l}\text { DII Score Tertile } 2 \\
(-1.3 \text { to } 4.5)(n=73)\end{array}$ & $\begin{array}{l}\text { DII Score Tertile } 3 \\
(>4.5)(n=74)\end{array}$ & & & \\
\hline \multicolumn{7}{|l|}{ VAS } \\
\hline Model 0 & Ref. & $1.19(0.66-1.75)$ & $1.33(0.88-2.05)$ & 0.17 & 1.22 & $(0.71-1.91)$ \\
\hline Model I & Ref. & $1.05(0.72-1.87)$ & $1.55(1.04-2.31)$ & 0.04 & 1.48 & $(1.26-2.02)$ \\
\hline \multicolumn{7}{|l|}{ WOMAC } \\
\hline Model 0 & Ref. & $1.09(0.59-1.46)$ & $1.59(0.77-1.99)$ & 0.78 & 1.40 & $(0.91-1.61)$ \\
\hline Model I & Ref. & $1.18(0.48-1.84)$ & $1.63(0.61-2.13)$ & 0.09 & 1.52 & $(0.58-1.90)$ \\
\hline
\end{tabular}

Model 0, Crude; Model I, Adjusted for age, sex, body mass index and physical activity

VAS was converted to tertiles, considering the first tertile (VAS $<4 \mathrm{~cm}$ ) as mild, second tertile $(4 \mathrm{~cm} \leq$ VAS $\leq 7 \mathrm{~cm})$ as moderate, and the last tertile (VAS $>7 \mathrm{~cm})$ as severe [40]. WOMAC was converted to tertiles, considering the first tertile (WOMAC $<24$ points) and second tertile $(25 \mathrm{~cm} \leq$ WOMAC $\leq 48 \mathrm{~cm})$, and the last tertile (WOMAC $>48 \mathrm{~cm}$ )

DII dietary inflammatory index, VAS visual analogue scale, WOMAC Western Ontario and McMaster Index

${ }^{a}$ Logistic regression analysis 
DII score in comparison with the lowest tertile (OR tertile 3 vs. $1=1.55$, 95\% CI 1.04-2.31; P trend $=0.04$ ).

\section{Discussion}

The present study found that consuming a diet with higher DII score was related with higher pain intensity and lower QOL. After adjusting for potential covariates, KOA patients with the highest DII score had significantly higher VAS, and lower QOL in terms of PF, RP, SF and $\mathrm{P}$ scales and $\mathrm{PH}$ subscale. In this study, we observed that higher DII of diet was associated with $48 \%$ higher likelihood of having severe pain in patients with KOA. However DII score was not significantly associated with functional status measured by WOMAC the patients. These findings reinforce the rising body of evidences on the promising role of foods with lower inflammatory capacity in decreasing the odds of severe symptoms of KOA.

The effect of diet on the inflammatory markers such as CRP has been well recognized in numerous observational investigations, with utmost investigations indicating that healthier diets may be related with lesser inflammation [25-28]. In recent years, the role of systemic low-grade inflammation in the pathogenesis of OA has been wellrecognized [29]. In patients with OA, upper systemic concentrations of pro-inflammatory cytokines are linked with further pain and dysfunction [30]. Distribution of these cytokines from the synovial fluid into the cartilage tissue plays a key role in cartilage matrix defect by arousing chondrocyte catabolic pathway and obstructing anabolic pathway [31]. Chronic low-grade inflammation may be a main stimulus of continuing joint degeneracy, therefore disturbing cartilages and bones structures characteristic for KOA. Additionally, inflammatory cytokines can induce muscle dysfunction and decrease the pain threshold [32].

Studies investigating the relationship between DII and $\mathrm{OA}$ are rare. Along with the results of this study, in A large cross-sectional study, Veronese et al. [33] showed that higher DII is linearly associated to increased prevalence of radiographic symptomatic KOA. Subjects with the highest DII score had a significantly increased occurrence of radiographic symptomatic KOA by almost $40 \%$. Furthermore, it has been found that extra-cellular matrix alterations and breakdown products may increase the inflammation and cartilage damage [34]. So it is probable these alterations play a further role in the association between DII and KOA. A pro-inflammatory dietary pattern can further stimulate these processes. It has also been shown that the DII has a reverse relationship with healthy dietary patterns (such as the Mediterranean diet) that can protect against KOA [35].
To our knowledge, our study is the first that examined the relationship between DII score and scales and subscales of QOL. However, the association of systemic inflammation with high levels of inflammatory and pro-inflammatory cytokines with reduced QOL in general and specific components including pain, physical and emotional health have been shown in previous studies [36-39].

\section{Conclusion}

In conclusion, DII score was associated with pain intensity (VAS) and PF, RP, and P scales and PH subscales of QOL in patients with KOA with and without adjustment for age, sex, BMI and physical activity. Higher DII in the diets was related with higher odds of severe pain in patients with KOA.

\section{Limitation}

First, we can't conclude confident cause and effect associations because of the cross-sectional design of the study. Relatively small sample size in second limitation. Third, the possibility of recall bias should be considered. Fourth, in our study, data are available on 29 of the 45 food parameters; lack of data on the residual food parameters intake is a limitation. Fifth, self-reported dietary approaches such as FFQ are exposed to bias, the incapability to quantity this bias and adjust for it is a limitation.

\section{Supplementary information}

Supplementary information accompanies this paper at https://doi. org/10.1186/s13104-020-05277-x.

Additional file 1. Standardized regression coefficients (B) and their standard error (SE) and $p$ value of the association between DII score and pain intensity, functional status and quality of life in patients with knee osteoarthritis.

\section{Abbreviations}

BMI: body mass index; Cl: confidence interval; DII: dietary inflammatory index; EW: emotional wellbeing; EF: energy/fatigue; FFQ: food frequency questionnaire; GH: general health; KOA: knee osteoarthritis; OA: osteoarthritis; P: pain; PF: physical function; QOL: quality of life; RE: role limitation due to emotional problems; RP: role limitation due to physical health; SF: social function; SD: standard deviation; VAS: visual analogue scale; WOMAC: Western Ontario and McMaster Universities Osteoarthritis Index.

\section{Acknowledgements}

We are thankful to all of the participants who helped us to conduct this study. The authors would like to acknowledge the staff of the Physical Medicine and Rehabilitation Research Center of Tabriz University of Medical Sciences for their support with this study. The results presented in this article were a part of an MD thesis by M.Rasouli.

\section{Authors' contributions}

$V T$, ND provided important contributions to the design of the study; ND, MR and DA to acquisition, analysis, or interpretation of data for the work; VT, ND, $\mathrm{MH}$ to drafting the work or revising it critically. All authors read and approved the final manuscript. 


\section{Funding}

There was no external funding resource for this study.

\section{Availability of data and materials}

All the necessary data are presented herewith. However if needed, raw data on excel format can be availed on reasonable request from the corresponding author.

\section{Ethics approval and consent to participate}

At the start of the investigation, the purposes and methods of the project were clarified completely for each participant, and written informed consent was acquired from all subjects. This study was approved by the Ethics Committee of the Research Vice-Chancellor of Tabriz University of Medical Sciences (IR.TBZMED.REC.1395.12.90).

\section{Consent for publication}

Not applicable.

\section{Competing interests}

None declared.

\section{Author details}

1 Physical Medicine and Rehabilitation Research Center, Aging Research Institute, Tabriz University of Medical Science, Azadi Ave., Tabriz, Iran. ${ }^{2}$ Palliative Care Medicine Department, Faculty of Medicine, Tabriz University of Medical Sciences, Tabriz, Iran. ${ }^{3}$ Faculty of Medicine, Tabriz University of Medical Sciences, Tabriz, Iran. ${ }^{4}$ Department of Biology, School of Arts and Sciences, Utica College, Utica, USA.

Received: 27 June 2020 Accepted: 9 September 2020

Published online: 21 September 2020

\section{References}

1. Van Schoor N, Smit J, Twisk J, Lips P. Impact of vertebral deformities, osteoarthritis, and other chronic diseases on quality of life: a populationbased study. Osteoporosis Int. 2005;16(7):749-56.

2. Goldring MB, Otero M. Inflammation in osteoarthritis. Curr Opin Rheumatol. 2011;23(5):471-8.

3. Sellam J, Berenbaum F. The role of synovitis in pathophysiology and clinical symptoms of osteoarthritis. Nat Rev Rheumatol. 2010;6(11):625-35.

4. Esmaillzadeh A, Kimiagar M, Mehrabi Y, Azadbakht L, Hu FB, Willett WC. Dietary patterns and markers of systemic inflammation among Iranian women. J Nutr. 2007;137(4):992-8.

5. Sureda A, Bibiloni MDM, Julibert A, Bouzas C, Argelich E, Llompart I, et al. Adherence to the mediterranean diet and inflammatory markers. Nutrients. 2018;10(1):62.

6. Dahan S, Segal Y, Shoenfeld Y. Dietary factors in rheumatic autoimmune diseases: a recipe for therapy? Nat Rev Rheumatol. 2017;13(6):348.

7. Cutolo M, Nikiphorou E. Don't neglect nutrition in rheumatoid arthritis! RMD open. 2018;4:1.

8. Gabriele M, Pucci L. Diet bioactive compounds: Implications for oxidative stress and inflammation in the vascular system. Endoc Metab Immune. 2017;17(4):264-75.

9. Shivappa N, Prizment AE, Blair CK, Jacobs DR Jr, Steck SE, Hebert JR. Dietary inflammatory index and risk of colorectal cancer in the lowa Women's Health Study. Cancer Epidemiol Biomarkers. 2014;23(11):2383-92.

10. Shivappa N, Hebert JR, Marcos A, Diaz LE, Gomez S, Nova E, et al. Association between dietary inflammatory index and inflammatory markers in the HELENA study. Mol Nutr Food Res. 2017:61:6.

11. Na W, Kim M, Sohn C. Dietary inflammatory index and its relationship with high-sensitivity $C$-reactive protein in Korean: data from the health examinee cohort. J Clin Biochem Nutr. 2018;62(1):83-8.

12. Shivappa N, Steck SE, Hurley TG, Hussey JR, Ma Y, Ockene IS, et al. A population-based dietary inflammatory index predicts levels of C-reactive protein in the Seasonal Variation of Blood Cholesterol Study (SEASONS). Public Health Nutr. 2014;17(8):1825-33.

13. Altman R, Asch E, Bloch D, Bole G, Borenstein D, Brandt K, et al. Diagnostic and Therapeutic Criteria Committee of the American Rheumatism Association. Development of criteria for the classification and reporting of osteoarthritis: classification of osteoarthritis of the knee. Arthritis Rheum. 1986;29(8):1039-49.

14. Mirmiran P, HoseiniEsfahani F, Azizi F. To assess the relative validity and reliability of food frequency questionnaires to assess food intakes: Tehran Lipid and Glucose Study [in persian]. IJDLD. 2010;9(2):185-97.

15. Willett WC, Howe GR, Kushi LH. Adjustment for total energy intake in epidemiologic studies. Am J Clin Nutr. 1997;65(4):1220S-8S.

16. Shivappa N, Steck SE, Hurley TG, Hussey JR, Hébert JR. Designing and developing a literature-derived, population-based dietary inflammatory index. Public Health Nutr. 2014;17(08):1689-96.

17. Cho S-H, Choi M, Lee J, Cho H. Relationship between Expectations Regarding Aging and Physical Activity among Middle Aged Adults in Urban Areas: Based on the Pender's Health Promotion Model. J Korean Acad Nurs. 2015:45:1.

18. Vasheghani-Farahani A, Tahmasbi M, Asheri H, Ashraf H, Nedjat S, Kordi R. The Persian, last 7-day, long form of the International Physical Activity Questionnaire: translation and validation study. Asian J Sports Med. 2011;2(2):106.

19. Hawker GA, Mian S, Kendzerska T, French M. Measures of adult pain: visual analog scale for pain (vas pain), numeric rating scale for pain (nrs pain), mcgill pain questionnaire (mpq), short-form mcgill pain questionnaire (sf-mpq), chronic pain grade scale (cpgs), short form-36 bodily pain scale (sf-36 bps), and measure of intermittent and constant osteoarthritis pain (icoap). Arthritis Care Res. 2011;63(S11):S240-52.

20. American Callege of Rheumatology. Western Ontario and McMaster Universities Osteoarthritis Index (WOMAC). https://www.rheumatology.org/ Practice/Clinical/Clinicianresearchers/Outcomes_Instrumentation/Weste rn_Ontario_and_McMaster_Universities_Osteoarthritis_Index_\%28WOM AC\%29/.

21. Tepper S, Hochberg MC. Factors associated with hip osteoarthritis: data from the First National Health and Nutrition Examination Survey (NHANES-I). Am J Epidemiol. 1993;137(10):1081-8.

22. Eftekharsadat B, Niknejad-Hosseyni SH, Babaei-Ghazani A, Toopchizadeh $V$, Sadeghi $H$. Reliability and validity of Persian version of Western Ontario and McMaster Universities Osteoarthritis index in knee osteoarthritis. J Anal Res Clin Med. 2015;3(3):170-7.

23. Montazeri A, Goshtasebi A, Vahdaninia M, Gandek B. The Short Form Health Survey (SF-36): translation and validation study of the Iranian version. Qual Life Res. 2005;14(3):875-82.

24. Schumacker RE, Lomax RG. A beginner's guide to structural equation modeling. 2nd ed. New Jersey London: Lawrence Erlbaum Associates Publishers; 2004

25. Fargnoli JL, Fung TT, Olenczuk DM, Chamberland JP, Hu FB, Mantzoros CS. Adherence to healthy eating patterns is associated with higher circulating total and high-molecular-weight adiponectin and lower resistin concentrations in women from the Nurses' Health Study. Am J Clin Nutr. 2008:88(5):1213-24.

26. Monfort-Pires M, Folchetti LD, Previdelli AN, Siqueira-Catania A, de Barros $C R$, Ferreira SR. Healthy Eating Index is associated with certain markers of inflammation and insulin resistance but not with lipid profile in individuals at cardiometabolic risk. Appl Physiol Nutr Metab. 2014;39(4):497-502.

27. Park KH, Zaichenko L, Peter P, Davis CR, Crowell JA, Mantzoros CS. Diet quality is associated with circulating C-reactive protein but not irisin levels in humans. Metabolism. 2014;63(2):233-41.

28. Fung TT, McCullough ML, Newby PK, Manson JE, Meigs JB, Rifai N, et al. Diet-quality scores and plasma concentrations of markers of inflammation and endothelial dysfunction. Am J Clin Nutr. 2005;82(1):163-73.

29. Berenbaum F. Osteoarthritis as an inflammatory disease (osteoarthritis is not osteoarthrosis!). Osteoarthritis Cartilage. 2013;21(1):16-21.

30. Penninx BW, Abbas H, Ambrosius W, Nicklas BJ, Davis C, Messier SP, et al. Inflammatory markers and physical function among older adults with knee osteoarthritis. J Rheumatol. 2004;31(10):2027-31.

31. Loeser RF, Goldring SR, Scanzello CR, Goldring MB. Osteoarthritis: a disease of the joint as an organ. Arthritis Rheum. 2012;64(6):1697-707.

32. Hunter DJ, McDougall JJ, Keefe FJ. The symptoms of osteoarthritis and the genesis of pain. Med Clin North Am. 2009;93(1):83-100.

33. Veronese N, Shivappa N, Stubbs B, Smith T, Hebert JR, Cooper C, et al. The relationship between the dietary inflammatory index and prevalence of radiographic symptomatic osteoarthritis: data from the Osteoarthritis Initiative. Eur J Nutr. 2017:58(1):253-60. 
34. Homandberg GA, Hui F. Association of proteoglycan degradation with catabolic cytokine and stromelysin release from cartilage cultured with fibronectin fragments. Arch Biochem Biophys. 1996;334(2):325-31.

35. Veronese N, Stubbs B, Noale M, Solmi M, Luchini C, Smith TO, et al. Adherence to a Mediterranean diet is associated with lower prevalence of osteoarthritis: data from the osteoarthritis initiative. Clin Nutr. 2017;36(6):1609-14

36. Robinson SM, Rasch S, Beer S, Valantiene I, Mickevicius A, Schlaipfer E, et al. Systemic inflammation contributes to impairment of quality of life in chronic pancreatitis. Sci Rep. 2019;9(1):7318.

37. Li L, Chan SL, Mo F, Hui EP, Koh J, Chan AKC, et al. Correlations of health-related quality of life with serum inflammatory indicators IL-8 and $\mathrm{mIBI}$ in patients with hepatocellular carcinoma. Cancer Manag Res. 2019;11:2719-27.

38. Dinh KM, Kaspersen KA, Mikkelsen S, Pedersen OB, Petersen MS, Thorner LW, et al. Low-grade inflammation is negatively associated with physical
Health-Related Quality of Life in healthy individuals: results from The Danish Blood Donor Study (DBDS). PLoS ONE. 2019;14(3):e0214468.

39. Nowakowski AC, Graves KY, Sumerau JE. Mediation analysis of relationships between chronic inflammation and quality of life in older adults. Health Qual Life Outcomes. 2016;14:46.

40. Jensen MP, Smith DG, Ehde DM, Robinsin LR. Pain site and the effects of amputation pain: further clarification of the meaning of mild, moderate, and severe pain. Pain. 2001;91(3):317-22.

\section{Publisher's Note}

Springer Nature remains neutral with regard to jurisdictional claims in published maps and institutional affiliations.
Ready to submit your research? Choose BMC and benefit from:

- fast, convenient online submission

- thorough peer review by experienced researchers in your field

- rapid publication on acceptance

- support for research data, including large and complex data types

- gold Open Access which fosters wider collaboration and increased citations

- maximum visibility for your research: over $100 \mathrm{M}$ website views per year

At BMC, research is always in progress.

Learn more biomedcentral.com/submissions 